\title{
Coulisses
}

Revue de théâtre

\section{Poésie et philosophie dans Faust I de Goethe}

\section{Bénédicte Abraham}

\section{(2) OpenEdition}

\section{Journals}

Édition électronique

URL : https://journals.openedition.org/coulisses/348

DOI : $10.4000 /$ coulisses.348

ISSN : 2546-9460

\section{Éditeur}

Presses universitaires de Franche-Comté

\section{Édition imprimée}

Date de publication : 31 décembre 2011

Pagination : 105-114

ISBN : 978-2-84867-404-9

ISSN : 1150-594X

\section{Référence électronique}

Bénédicte Abraham, " Poésie et philosophie dans Faust / de Goethe », Coulisses [En ligne], 43 | Automne 2011, mis en ligne le 30 novembre 2016, consulté le 30 décembre 2022. URL : http:// journals.openedition.org/coulisses/348; DOI : https://doi.org/10.4000/coulisses.348 


\title{
Poésie et philosophie dans Faust I de Goethe
}

\author{
Bénédicte Abraham
}

1 L'interrogation qui porte sur l'alliance ou la mésalliance entre la philosophie et la poésie n'est pas nouvelle. Elle remonte à l'Antiquité, mais la fascination réciproque de ces deux champs du langage et de la pensée n'en est pas moins restée vive pour autant. Dans sa Poétique ${ }^{1}$, Aristote consacre plusieurs pages à la personne du poète dont il considère l'activité comme étant plus noble et plus philosophique que celle de l'historien. Ce qui constitue, aux yeux d'Aristote, la supériorité de la poésie sur l'histoire, c'est son caractère philosophique et sa tendance à exprimer l'universel, c'està-dire sa capacité à fournir des modèles. Platon chasse pourtant les poètes de sa cité idéale au livre III de la République et interdit aux gardiens-philosophes chargés d'administrer la cité de pratiquer la poésie au prétexte que la mimesis ne donne qu'un reflet déformé de la réalité sensible ${ }^{2}$. La disqualification des poètes par Platon est sévère et l'un des motifs de leur discrédit est la distance de leur propos par rapport à la réalité. Si la philosophie a souvent revendiqué sa suprématie spirituelle sur la poésie, la poésie a souvent affirmé sa puissance philosophique qui lui permet de dire des choses que la philosophie ne peut pas dire. La problématique d'une difficile articulation à trouver entre poésie et philosophie se retrouve sous la plume de Goethe et Schiller au sein de la correspondance assidue qu'ils entretiennent de 1794 à la mort de Schiller en 1805. Schiller en particulier, ressent douloureusement la peine qu'il éprouve à harmoniser ces deux champs du savoir et de la créativité et se plaint, dans plusieurs lettres à son ami, de la dissymétrie qui affecte la confrontation entre poésie et philosophie. La supériorité qu'il accorde à la poésie sur la philosophie, parce qu'elle est selon lui une activité noble et non prosaïque, rejoint, en certains points, la conception aristotélicienne ; il va même jusqu'à faire du poète le seul être véritablement humain et du philosophe une caricature du premier, comme il ressort d'une lettre qu'il écrit à Goethe le 7 janvier 1795: «[...] weil alle Natur nur Synthesis und alle Philosophie Antithesis ist [...] so viel ist indes gewiss, der Dichter ist der einzige wahre Mensch, und der beste Philosoph ist nur eine Karikatur gegen ihn $»^{3}$. Philosopher lui demande peu 
d'énergie et ne sollicite que la moitié de son être alors que les muses le vident de ses forces :

Ich fürchte, ich muss die lebhaften Bewegungen büßen, in die mein Poetisieren mich versetzte. Zum Philosophieren ist schon der halbe Mensch genug, und die andere Hälfte kann ausruhen ; aber die Musen saugen einen aus ${ }^{4}$.

Que la philosophie soit pour Schiller une activité prosaïque, c'est une idée qu'il exprime dans une autre lettre à Goethe datée du 17 décembre 1795 :

Ich habe mich lange nicht so prosaisch gefühlt als in diesen Tagen und es ist hohe Zeit, dass ich für eine Weile die philosophische Bude schließe. Das Herz tastet nach einem betastlichen Objekt ${ }^{5}$.

3 Goethe insiste, quant à lui, plutôt que sur la disjonction douloureuse entre poésie et philosophie, sur leur nécessaire harmonisation, qui est un élément constitutif de l'être humain véritablement cultivé : «[...] nur in einer gewissen Verbindung der Philosophie und Poesie bestehe die wahre Bildung ", écrit-il à Schiller"

4 Évoquant la gestation de sa tragédie Faust, Goethe fait part, dans une lettre à son $\mathrm{ami}^{7}$, de l'équilibre qu'il n'a eu de cesse de rechercher entre la pensée et l'expressivité. Ce sont pourtant les philosophes de son époque qui semblent avoir attendu avec l'impatience la plus vive la parution du Faust, comme le mentionne Schiller dans une lettre du 16 mars 1801: "Viel Glück zu den Fortschritten im "Faust", auf den die hiesigen Philosophen ganz unaussprechlich gespannt sind $»^{8}$. Comme le rappelle Richard Baum dans un article consacré à l'idée que Goethe se fait de la poésie ${ }^{9}$, l'écrivain et penseur a cherché précisément à définir l'essence de la poésie en l'opposant à la philosophie et à la mystique ${ }^{10}$. On peut donc en toute légitimité s'interroger sur la place respective de la philosophie et de la poésie dans la tragédie de Goethe et réfléchir à leur articulation et leur expression dans une pièce qui, en dernière instance, relève du drame au sens étymologique tel que le définit Aristote ${ }^{11}$, et met donc à l'honneur l'action plus que le langage ou la pensée. Peut-être Goethe a-t-il à cœur, en cherchant à harmoniser dans Faust la poésie et la philosophie, de dépasser le dualisme dans lequel elles sont enfermées en faisant de son œuvre maîtresse le chefd'œuvre de la poésie pensante et de la philosophie expressive?

\section{La voix sacrée du poète}

5 Considérons tout d'abord que la parole est d'emblée donnée au poète: la tragédie s'ouvre sur une dédicace du poète qui se présente sous la forme d'un poème en stances de quatre strophes dans lequel l'auteur fait part des difficultés rencontrées lors de la création de son œuvre et évoque avec nostalgie et douleur, sur un ton élégiaque, ses jeunes années où il s'était attelé à la tâche ardue d'écrire un drame qu'il reprend aujourd'hui avec peine. Il y est question du chant du poète par lequel il exprime sa douleur de n'être peut-être pas compris de la foule :

Mein Leid ertönt der unbekannten Menge,

Ihr Beifall selbst macht meinem Herzen bang,

Und was sich sonst an meinem Lied erfreuet,

Wenn es noch lebt, irrt in der Welt zerstreuet ${ }^{12}$.

6 C'est donc la voix du poète qui s'élève pour initier le drame. On la retrouve dans la scène suivante, «Prolog im Theater » («Prologue sur le théâtre »), matérialisée cette fois par le personnage du poète siégeant sur scène aux côtés du directeur du théâtre et 
du bouffon. Le «je » lyrique qui s'était exprimé dans la dédicace fait ici l'objet d'une dissociation matérielle entre trois personnages qui reprennent chacun à leur compte les questions soulevées en préambule par le seul poète et qui concernaient les rapports entre le poète et ses personnages, le poète et son public. Deux conceptions antithétiques s'opposent dans ce prologue : il s'agit de celle du poète, épris d'absolu et d'idéalisme et de celle du directeur du théâtre, âpre au gain et soucieux de la réussite sociale et matérielle du projet ${ }^{13}$. Sans doute les réparties du directeur du théâtre sontelles destinées, dans leur matérialisme outrancier, à souligner favorablement les ambitions éthérées du poète qui, tel un Dieu, crée et assure la pérennité de l'Olympe ${ }^{14}$. Il ressort du dernier vers du passage que l'épiphanie du génie humain est assurée par le poète. La poésie est déclarée bien supérieure à toutes les formes de langage et de pensée, elle a vocation d'absolu et confine au sacré.

\section{La mélancolique et élégiaque méditation de Faust sur le sens}

7 La tragédie proprement dite le confirme en s'ouvrant sur le savant Faust qui médite le désastre du sens et déplore l'impouvoir foncier du langage. La vanité du savoir le rend mélancolique et fait surgir en lui des idées de suicide. La première des facultés étudiées qu'il évoque en un accord initial chargé de profonde mélancolie est d'ailleurs la philosophie ${ }^{15}$. Le monologue entier de Faust est constitué de trois raisonnements antithétiques par lesquels le savant oppose ses longues et savantes études à son faible savoir ${ }^{16}$ et il achève sa tirade par une condamnation méprisante du langage ${ }^{17}$ qui n'est pas sans rappeler la haine de Herder pour ce que ce dernier appelait « la boutique de mots creux $»^{18}$, mais il s'apparente à un long poème et comme le lieu où le sujet vient porter plainte. Le premier monologue de Faust est tout à la fois une crise et un procès. La déception de Faust éprouvée face à l'impuissance du savoir et à la stérilité de la pensée fait alors place à une apostrophe à la lune, empreinte de lyrisme et de douceur élégiaque, où Faust se fait le chantre de la nature qui saura le guérir de ses tourments. Le ton, le rythme, le style et le vocabulaire deviennent tout autres ${ }^{19}$. Déçu par la science livresque, constatant non sans amertume qu'il a étudié la philosophie, mais qu'il ne philosophe pas, Faust se tourne alors vers la magie blanche, espérant trouver par la pratique magique un accès intime au monde et une harmonie entre microcosme et macrocosme, homme et univers ${ }^{20}$. Conçu à l'image des alchimistes et des philosophes mystiques de la Renaissance, Faust, s'adonnant à la pratique de la magie blanche, est davantage théosophe que philosophe, dans la mesure où il cherche par l'intuition et l'extase, bien plus que par la connaissance scientifique ou le raisonnement philosophique, à s'élever vers une vision radieuse du monde et aspire à une connaissance mystique et non raisonnée de la Nature. La formule magique par laquelle Faust invoque l'Esprit de la terre vient rappeler la puissance expressive du langage et de la poésie; Faust fait ici un usage poétique du langage ancré dans le sensible, la sensualité, le désir, qui n'est pas simple ornement ni simple jeu avec les mots, mais qui est motivé par l'invocation de l'Esprit de la terre auquel il s'adressera, comme en un hymne de gratitude, dans un second monologue dans la scène "Wald und Höhle " $^{21}$ ( Forêt et caverne »). À cet usage poétique et presque extatique du langage, Goethe oppose à dessein l'admiration naïve de Wagner envers un usage rhétorique du langage et, soulignant le prosaïsme du personnage, lui donne le rôle d'un faire-valoir du 
théosophe épris d'absolu qu'est Faust ${ }^{22}$. La seule parole de Wagner que l'on pourrait, dans cet échange avec Faust, juger poétique en raison du recours à la figure du chiasme, n'est en fait qu'une pâle et scolaire imitation d'Hippocrate, fort éloignée du génie poétique créateur et inspiré :

Ach Gott ! die Kunst ist lang !

Und kurz ist unser Leben ${ }^{23}$.

Mon Dieu! Que l'art est long et que la vie est brève $e^{24}$ !

8 C'est dans la rencontre avec son famulus, qui n'est finalement qu'une caricature de luimême ou de son moi qu'il voudrait déjà antérieur, que Faust prend toute la mesure de sa crise existentielle et du drame de son identité. Le drame de Faust est celui de la connaissance doublé d'un drame existentiel, c'est le drame universel du sujet malheureux et scindé qui recherche la réconciliation entre le monde de la matière et le monde de l'esprit. La tragédie de Goethe est sous-tendue par un questionnement philosophique : le souci de Faust de mener une enquête sur soi, ses limitations et sur la nature du bonheur possible à l'homme. C'est un drame existentiel plus que métaphysique, en dépit du «Prologue au Ciel » qui met en scène la confrontation manichéenne entre Dieu et le Diable :

Il ne faut pas oublier que la tragédie de Faust s'ouvre en cette nuit du samedi de Pâques où Dieu est mort. Faust est donc conduit à un insupportable « connais-toi toi-même » et découvre son identité comme une innommable vérité. Sa subjectivité naguère déchaînée et triomphante n'a d'autre issue que ce radicalisme de l'anéantissement ${ }^{25}$.

Dans sa recherche d'une vérité sur lui-même et sur les conditions du bonheur terrestre, dans sa dénonciation du potentiel mensonger du langage, Faust est engagé sur une voie philosophique dont le mode d'expression privilégié reste, en dernière instance, le dialogue. Le geste fondateur de la philosophie procède d'une exclusion $\mathrm{du}$ «je » au profit du couple constitué du questionnement et de ses réponses. La crise existentielle de Faust réclame des actes et une résolution que seule la forme dramatique est à même de signifier. Aussi la tragédie de Goethe s'apparente-t-elle à un poème philosophique en acte et en actes; l'action est jugée bien supérieure au langage dans ce passage de la scène « Studierzimmer » («Cabinet de travail ») où Faust s'apprête à traduire l'Évangile selon saint Jean ${ }^{26}$. Dans ce passage, Faust se fait poète et philosophe, bien plus que théologien. Il renie, du reste, la théologie qu'il regrette d'avoir étudiée ${ }^{27}$. Il modifie en profondeur le sens du texte biblique, fait œuvre de poète en montrant ici une parole qui bouge et cherche sa place, une parole qui existe de se chercher et subsiste de se questionner ${ }^{28}$, comme lui. Le travail du poète est de restituer à la langue sa matérialité, sa densité, son épaisseur ${ }^{29}$ et sa force, ce que Faust cherche à faire dans ce passage en cherchant la formule d'énergie, le souffle, le son qui le satisfera et qui explose au vers 1237 dans le terme «action» («Die Tat»). La poésie rejoint la philosophie dans la mesure où toutes deux sont des activités philologiques, activités de lecture et d'écriture, lecture critique et réécriture de sa propre vie pour Faust. Faust va penser sa vie en mouvement, en se mettant en mouvement aux côtés de Méphistophélès et se faire progressivement l'artisan philosophe de sa nouvelle existence. Après la poétique du désaccord fondamental avec lui-même exprimé dans le cri déchirant suggérant sa scission intérieure «Zwei Seelen wohnen ach! in meiner Brust » (« Deux âmes, hélas! habitent en ma poitrine ») de la scène « Vor dem Tor »" («Devant la porte de la ville »), la question centrale du drame va s'énoncer de la manière suivante: comment réaccorder l'être humain, au double sens musical et conceptuel du verbe 
"réaccorder", comment réaccorder la pensée et le désir, le discours conceptuel philosophique auquel Faust est si exercé et le discours mythique poétique dont il est l'emblème ? La vie de Faust, dont le mouvement va de la quête du savoir à la quête du sens, devient poésie pensante, se fait "poésophie », c'est-à-dire articulation intime entre poésie et philosophie et acquisition d'une sagesse de vie par la parole poétique dont Gretchen est l'emblème.

Goethe insiste dans son drame sur une poétique du contraste qui suggère la lente et difficile réunification à lui-même de Faust. Deux personnages sont convoqués pour suggérer la crise identitaire de Faust : il s'agit de Méphisto et de Gretchen. Méphisto représente une sorte de projection abjecte de Faust, son mauvais " moi », son double malfaisant, hétérogène et démoniaque, l'altérité qu'il a refoulée ${ }^{32}$. C'est dans le rapport à l'autre que se définit le propre, il ne peut $\mathrm{y}$ avoir d'identité que là où il $\mathrm{y}$ a différence. On ne se connaît soi-même que par la relation, le rapport établi, comme en amour. Méphisto souligne la faille identitaire de Faust, dans laquelle il s'engouffre d'ailleurs dans l'espoir de triompher dans le cadre du pari conclu avec le Seigneur. C'est précisément parce que Faust a une double nature, possède deux âmes, l'une noble, attirée par les hautes sphères de l'esprit et de la science, l'autre vile, sensible aux jouissances bassement terrestres, que Méphisto est détenteur d'un pouvoir sur Faust qu'il ne peut avoir sur Gretchen qui est une nature entière, simple, non scindée et dont il dira justement: «Über die hab' ich keine Gewalt » ${ }^{33}$ («Je n'ai pas de pouvoir sur elle »). Les deux thèmes principaux de la pièce sont ceux de l'amour et de la liberté. Faust est mis face à la liberté de choisir entre la voie du Bien ou celle du Mal, de répondre de ses actes en ayant éventuellement recours au repentir et à l'expiation de ses erreurs qui concernent essentiellement sa relation amoureuse à Gretchen. Pour signifier l'amour qui les lie, Goethe a d'ailleurs recours à une métaphore florale très signifiante et programmatique pour le reste de la tragédie; elle se situe dans la scène où Gretchen-Marguerite effeuille précisément la marguerite sous les yeux de Faust et, ce faisant, signifie, en détruisant progressivement la fleur, sa propre destruction à venir, rappelant aussi que le drame bourgeois dans lequel s'inscrit la tragédie de Marguerite ne laisse aucune possibilité d'issue heureuse à l'amour-passion ${ }^{34}$.

\section{Le personnage de Gretchen et la physique de la parole}

Gretchen se fait poète dans le passage où elle recourt à la métaphore qui est analogie et transposition, redistribution du sens de façon imagée. Le mot renvoie à l'idée, la fleur effeuillée renvoie à la virginité menacée. Cette approche métaphorique de l'expression de son amour est une façon pour Gretchen d'entrer en relation avec sa réalité à venir. Elle exprime métaphoriquement sa propre histoire. La métaphore est ici motivée et référentielle par rapport à l'histoire de Gretchen. La scène développe une esthétique de la poésie où prédomine la logique de l'analogie, de la comparaison, de la non-identité. Gretchen est sans doute, de tous les personnages de la pièce, celui dont le mode d'expression privilégié est le plus poétique, émotionnel, non réflexif ni discursif. À trois reprises $^{35}$, elle a recours au chant ou à la prière, c'est-à-dire au pouvoir incantatoire et consolateur de la parole poétique pour dissiper son malaise ou exprimer son amour. À la fin de la scène "Abend» («Le soir»), par exemple, où Faust et Méphisto s'introduisent dans la chambre de Gretchen à son insu pour y déposer le coffret à bijoux, cette dernière éprouve confusément un malaise à son retour, elle ressent la 
présence hostile du Diable pour lequel elle avouera son antipathie dans la scène "Marthens Garten » ${ }^{36}$ ("Le jardin de Marthe ») et entonne alors la ballade du roi de Thulé pour dissiper sa frayeur ${ }^{37}$. La puissance de la poésie réside en ce qu'elle peut dire des choses que la philosophie est impuissante à dire comme la peur, l'appréhension ou encore l'amour. Le mode d'accès à l'être et à la connaissance chez Gretchen est beaucoup plus intuitif que discursif. Il y a identité chez Gretchen de l'objet et du sujet. La poésie est "mesurée », elle régule, cadastre les émotions violentes éprouvées. Gretchen est présente au réel par le chant, elle est « toute ", c'est-à-dire qu'elle est dans l'existence grâce à l'essence de la parole. Les passages où Gretchen s'exprime par des chants appellent une typographie particulière, une dimension plastique, rythmique et immédiatement sensible, c'est-à-dire une physique de la parole. Le sens, en même temps qu'il est formel, est aussi, dans ces passages, existentiel. La poésie à laquelle Gretchen a recours produit du sens. La "Stimmung », qui évoque une tonalité affective inséparable de l'atmosphère, qui renvoie à l'impression qui se dégage de la configuration des choses, de la disposition des mots, n'est précisément pas l'expression d'un sujet, logique et raisonnée. Gretchen est dans le domaine de la signifiance poétique et non de la signification conceptuelle. Gretchen est une enfant qui vit dans une sorte d'enfance du langage où il n'y a pas de moyen de communiquer conceptuel. Elle redécrit le monde au lieu de le décrire. La poésie demande d'entendre avant de comprendre, de comprendre en prononçant. Le rôle des poètes n'est-il pas de retrouver l'essence des mots, des silences? Sur ce point, elle est l'exact opposé de Faust qui incarne la réflexivité angoissée et la conceptualisation aride. Goethe semble avoir travaillé ces deux personnages en contraste, comme certaines scènes le soulignent. C'est le cas, par exemple, de la scène " Marthens Garten » (" Le jardin de Marthe ») dans laquelle s'engage entre Gretchen et Faust une conversation au sujet de la religion. Faust se présente comme un libre penseur, à peine encore attaché au christianisme teinté de sentimentalisme de son enfance. En ce sens, Faust, détourné du sacré et du religieux n'est pas un être poétique. Il y a, par ailleurs, dans la tragédie de Goethe, quelques scènes comiques ou burlesques qui viennent relativiser la figure et le pouvoir du Diable, mieux que ne sauraient le faire des réflexions philosophiques ou théologiques.

\section{Conclusion}

De manière générale, la tragédie de Goethe est sous-tendue par une opposition entre un discours poétique et un discours non-poétique ou prosaïque que viennent incarner quelques personnages comme Dame Marthe, veuve joyeuse et cupide, ou encore Wagner, fermé à toute dimension surnaturelle de la vie, comme on le constate dans la scène "Vor dem Tor» ("Porte devant la ville»), où il ne voit dans le barbet qui s'attache à Faust à la fin de leur promenade pascale rien d'autre qu'un vulgaire barbet, là où Faust pressent une force surnaturelle et énigmatique. La pièce de Goethe contient de nombreux passages à caractère lyrique et quelques dialogues à caractère philosophique ; il n'en reste pas moins que Goethe a choisi la théâtralisation et la forme du drame pour, in fine, donner forme au mythe de Faust. C'est-à-dire qu'il a voulu privilégier le sens de l'action. Ce sont le drame et l'action qui affirment leur primauté sur la philosophie et la poésie. Certes, il mélange les genres - lyrique et dramatique -, ne serait-ce qu'en écrivant toute sa pièce en vers (à l'exception de la scène en prose intitulée « Trüber Tag. Feld. » / " Jour sombre. La campagne »), mais ses références sont néanmoins essentiellement théâtrales, allant de la tragédie antique avec l'insertion des 
prologues et la présence de chœurs qui relèvent de la tradition antique, au drame shakespearien en intégrant de nombreuses références intertextuelles à quelques-unes des pièces de l'auteur anglais, comme Macbeth avec la présence des sorcières dans les scènes "Hexenküche " ("Cuisine de sorcière ») et "Walpurgisnacht» (« Nuit de Walpurgis »), ou encore au drame bourgeois qui est la référence sous-jacente de la tragédie dite « de Marguerite ». Ainsi, la tragédie de Goethe met en évidence la fracture et l'harmonie difficile entre poésie et philosophie incarnée par le personnage éponyme de la pièce, mais éclatée aussi selon les personnages qui forment la constellation principale, à savoir Gretchen et Méphisto. Si le langage est l'instrument privilégié de la poésie et de la philosophie, la pièce de Goethe est sous-tendue par une réflexion sur l'efficience du langage et parsemée de passages dans lesquels une critique du langage rhétorique et mensonger - est à l'œuvre. Faust dira à Méphisto qu'il est un menteur, doublé d'un sophiste ${ }^{38}$, c'est-à-dire un ennemi du vrai philosophe. Lui-même ne sera pas à l'abri d'une telle accusation, car il va, auprès de Gretchen, faire un usage abusif du langage et faire autant de beaux mots comme amant que comme savant, ce que ne manque pas de lui rappeler le Diable dans la seconde scène intitulée "Straße » ${ }^{39}$ (« Une rue »). La tragédie de Goethe met en lumière la crise d'un sujet en quête de sens, la crise de la subjectivité comme plénitude psychologique à exprimer. La philosophie dans la pièce, le "vouloir dire ", ne domine pas la poésie et c'est pour finir dans le «vouloir agir ", dans l'action dramatique que Faust va entrevoir une issue à son déchirement intérieur initial. Élevant son personnage au rang de figure mythique qui cherche la réunification à lui-même par l'affirmation du désir pensant et de la pensée désirante, Goethe concourt au développement de l'idée d'une pensée poétique en action.

\section{NOTES}

1. Aristote, Poétique [Introduction, traduction nouvelle et annotation de Michel Magnien], Paris, Le Livre de poche, « Classiques », 1990, p. 98.

2. Platon, République, « livre III », cité par Michel Magnien dans Aristote, Poétique, ibid. p. 141.

3. Ibid. p. 55.

4. Lettre à Goethe du 29 août 1795, ibid. p. 103 : «Je crains de devoir expier les mouvements pleins de vie dans lesquels m'a jeté mon activité poétique. Pour philosopher, il n'est besoin que d'une moitié d'homme, quand l'autre moitié peut se reposer; mais les muses vous sucent jusqu'à la moelle. » [Traduction B. A.]

5. Lettre à Goethe du 17 décembre 1795, ibid.p. 138-139: «Cela fait longtemps que je ne me suis senti aussi prosaïque que ces derniers jours et il est grand temps que je ferme pour un moment la baraque philosophique. Mon cœur aspire à un objet plus palpable. » [Traduction B. A.]

6. «La véritable formation de l'individu ne consiste qu'en un certain lien établi entre philosophie et poésie » [Traduction B. A.], Lettre à Schiller du 9 août 1797, ibid. p. 388.

7. Lettre à Schiller du 27 juin 1797, op. cit., p. 366-367.

8. «Bonne chance pour avancer dans Faust, que les philosophes attendent ici avec une curiosité indicible » [Traduction B. A.], Lettre à Goethe du 16 mars 1801, op. cit., p. 360. 
9. Richard Baum, «Dichtung und Sprache: Goethes Vorstellung von Poesie ", in: Faust, modernisation d'un modèle, Paris, L'Harmattan, 2005, p. 95-120.

10. R. Baum, ibid. p. 95-96.

11. «[Homère et Aristophane] imitent tous deux des gens qui agissent et font quelque chose. Voilà pourquoi, selon certains, ces œuvres sont aussi appelées drames $[\delta \rho \alpha \mu \alpha \tau \alpha]$ : elles imitent des gens qui font quelque chose ", Poétique, op. cit., p. 88.

12. "Zueignung ", v. 21-24 : "C'est pour des inconnus que je souffre ou que j'aime ; / Quand leur foule applaudit, j'hésite, je ne sais. / De tous ceux que mon chant émouvait à la ronde, / Le peu qui vit encore est épars dans le monde. ", Goethe, Faust I et II, « dédicace » [Traduction par Jean Malaplate et présentation par Bernard Lortholary], Garnier-Flammarion, Paris 1984, p. 24. [C'est de cette édition que sont extraites toutes les traductions françaises du présent article.]

13. «Prolog im Theater », v. 37, 90 et 98.

14. Ibid., v. 59 ; v. 63-66 ; v. 156-157. [Traduction, p. 24, 26 et 28]

15. « Nacht » (« Nuit »), v. 354-357. [Traduction, p. 35]

16. Ibid., v. 358-359 et v. 364 .

17. Ibid., v. 385.

18. « Wortkrämerei ».

19. Ibid., v. 386-397. Notons au passage que l'invocation à la lune est un motif récurrent chez les poètes allemands préromantiques, ce qui souligne encore davantage le lyrisme du passage. [Traduction, p. 36]

20. Ibid., v. 377-383. [Traduction, p. 36]

21. « Wald und Höhle » (« Forêt et caverne »), v. 3217-3218.

22. « Nacht », v. 522-525; 546-547. [Traduction, p. 41]

23. Ibid., v. 558-559.

24. Ibid., p. 41.

25. Denise Blondeau, «Le poète et le diable, ou la révolution du langage poétique ", in : Faust, modernisation d'un modèle, op. cit., p. 65.

26. «Studierzimmer I » (« Cabinet de travail I ), v. 1224-1237. [Traduction, p. 63]

27. « Nacht », v. 356.

28. C'est aussi parce que sa modalité d'être au monde est interrogative et non assertive comme celle du Dieu du « Prologue au Ciel » que Faust est homme.

29. Richard Baum, op. cit., p. 117.

30. Notons ici que le «Ach!» devient l'emblème acoustique de la césure intérieure de Faust et que la poétique du désaccord est assurée par l'intonation du « Zwei ».

31. «Vor dem Tor » (« Devant la porte de la ville »), v. 1112.

32. À ce sujet, lire l'article de Denise Blondeau, op. cit., p. 65.

33. « Strasse » (« Une rue »), v. 2626.

34. « Garten » (« Un jardin »), v. 3179-3186. [Traduction, p. 147]

35. Dans les scènes «Abend » (« le soir »), «Gretchens Stube » («La chambre de Gretchen ») et « Zwinger» (« Le rempart»).

36. « Marthens Garten » (« Le jardin de Marthe »), v. 3472-3473.

37. Ibid., v. 2753-2760. [Traduction, p. 125-126]

38. Ibid., «Straße II », v. 3050.

39. Ibid., v. 3052-3054. 
INDEX

oeuvrecitee Faust I

Palavras-chave : poesia, filosofia

Mots-clés : poesie, philosophie

Palabras claves : poesia, filosofia

Keywords : poetry, philosophy

AUTEURS

BÉNÉDICTE ABRAHAM

Université de Franche-Comté

Maître de conférences HDR en langue et littérature germaniques 